\title{
CTNS Molecular Genetics Profile in a Portuguese Cystinosis Population
}

\author{
Filipa Ferreira ${ }^{*}$, Inês Leal' ${ }^{2}$, David Sousa², Teresa Costa ${ }^{3}$, Conceição Mota ${ }^{3}$, Ana Marta Gomes ${ }^{4}$, \\ Daniela Lopes, Maria do Carmo Macário5, Isabel Tavares', Helena Pinto ${ }^{6}$, \\ João Paulo Oliveira6,7,8, Rita Magriço' ${ }^{9}$, Célia Carmona1, Sónia Ramos1, \\ Raquel Neiva1, Ana Marcão ${ }^{1}$, Laura Vilarinho ${ }^{1,10}$
}

\author{
${ }^{1}$ Newborn Screening, Metabolism and Genetics Unit, Human Genetics Department, National Institute of Health Dr Ricardo Jorge, \\ Porto, Portugal \\ ${ }^{2}$ Centro Hospitalar Lisboa Norte, Hospital de Santa Maria, Lisboa, Portugal \\ ${ }^{3}$ Centro Hospitalar Universitário do Porto, Porto, Portugal \\ ${ }^{4}$ Centro Hospitalar Vila Nova de Gaia, Espinho, Portugal \\ ${ }^{5}$ Centro Hospitalar Universitário de Coimbra, CHUC, Coimbra, Portugal \\ ${ }^{6}$ Serviço de Genética Médica, Centro Hospitalar de São João, Porto, Portugal \\ ${ }^{7}$ Unidade de Genética, Faculdade de Medicina da Universidade do Porto, Porto, Portugal \\ ${ }^{8}$ I3S-Instituto de Investigação e Inovação em Saúde, Porto, Portugal \\ ${ }^{9}$ Serviço de Nefrologia do Hospital Garcia de Orta, Almada, Portugal \\ ${ }^{10}$ Research and Development Unit, Department of Human Genetics, National Institute of Health Dr Ricardo Jorge, Porto, Portugal \\ Email: *filipa.ferreira@insa.min-saude.pt
}

How to cite this paper: Ferreira, F., Leal, I., Sousa, D., Costa, T., Mota, C., Gomes, A.M., Lopes, D., do Carmo Macário, M., Tavares, I., Pinto, H., Oliveira, J.P., Magriço, R., Carmona, C., Ramos, S., Neiva, R., Marcão, A. and Vilarinho, L. (2018) CTNS Molecular Genetics Profile in a Portuguese Cystinosis Population. Open Journal of Genetics, 8, 91-100.

https://doi.org/10.4236/ojgen.2018.84008

Received: November 16, 2018

Accepted: December 25, 2018

Published: December 28, 2018

Copyright $\odot 2018$ by author(s) and Scientific Research Publishing Inc. This work is licensed under the Creative Commons Attribution International License (CC BY 4.0). http://creativecommons.org/licenses/by/4.0/

\section{Open Access}

\begin{abstract}
Background: Cystinosis is a multisystemic autosomal recessive deficiency of the lysosomal membrane transporter protein (cystinosin) caused by mutations in CTNS gene. Objective. This study summarizes the Portuguese experience in the diagnosis and management of patients with this rare disease over the past few years and reports recurrent mutations in the CTNS gene. Methods. Unrelated patients from different pediatric and adult hospitals all over Portugal with non-nephrotic proteinuria, hypercalciuria, hypokalemia impaired proximal reabsorption of amino acids, glycosuria and hypophosphatemia, suggestive of a Fanconi syndrome and ocular problems, were studied. Intra-leukocyte cystine levels were determined and molecular analysis was performed, to determine the presence or absence of the $57-\mathrm{kb}$ deletion in CTNS, followed by direct sequencing of the coding exons of CTNS. Results: From 1998 to 2017, twenty-one cystinotic patients were biochemically diagnosed. From the remaining seventeen (four deceased), eleven were studied for CTNS gene. Five out of eleven patients were homozygous for the 57-kb deletion $(10 / 22 ; 45.5 \%)$, and other five were compound heterozygous for this variant (15/22; 68.2\%). The other mutations found were p.Q128X (c.721 C>T; 2/22), p.S139F (c.755 C>T; 4/22) and c.18-21delGACT (p.T7FfsX7; 1/22). All
\end{abstract}


of these seventeen cystinotic patients are in treatment. Approximately $84 \%$ are adults, $16 \%$ are young children, and $54.5 \%$ are kidney transplant recipient. Conclusions. The authors would like to emphasize the importance of first screening for the $57-\mathrm{kb}$ deletion since it is very common in our population. This genetic study is the first in our country and it could be the basis for future genetic counseling in Portuguese population.

\section{Keywords}

Cystinosis, CTNS Gene, Mutational Spectrum, Kidney Failure, 57-kb Deletion

\section{Introduction}

Cystinosis (OMIM \#606272 ORPHA213) was first described in literature in 1903 [1] and is a rare autosomal recessive systemic lysosomal storage disease characterized by the abnormal accumulation of lysosomal cystine due to mutations in the cystinosin gene (CTNS) [2]. The CTNS gene is localized on human chromosome 17 p13 contains 12 exons, the first two of which are non-coding [2] [3] and the other ten encode a 367 amino acid protein named cystinosin. The incidence of this disease is estimated to be 1 in 100,000 - 200,000 live births, though exact numbers are difficult to obtain because the disease is often undiagnosed and/or misdiagnosed. Cystinosis is the most frequent cause of inherited Fanconi syndrome and should be considered the initial differential diagnosis. Nevertheless, the severity of Fanconi Syndrome associated with cystinosis requires rigorous treatment that is frequently complex [4]. Although the kidneys are initially affected during the first year of life, in most of the cases, other potentially affected organs include also eyes, thyroid, pancreas, gonads, muscles and CNS over time [3] [5]. The severity of the phenotype is correlated with the genotype. Accurate measurement of intracellular cystine content is obligatory for the diagnosis of cystinosis and still the cornerstone for both diagnosis and therapeutic monitoring of the disease [6].

The most commonly detected cystinosis mutation is a $57-\mathrm{kb}$ deletion that deletes the majority of CTNS gene [7] [8] and in general, is related with high doses of cystine in leukocytes. This deletion is present in almost $50 \%$ of all the Northern European and North American origin [3] [7]-[13]. However, outside this geographical distribution, the mutation is almost completely absent, especially in the Middle East [14] [15] [16], therefore migration and/or ethnicity could be the explanation for this fact as well as the concept of possible other founder mutation from those regions [3]. Since the cloning of CTNS gene, over 200 pathogenic mutations have been reported in different ethnic groups, including mutations in the promoter region, missense, nonsense, deletion, insertion and splice mutations(http://www.hgmd.org/) [13] [17].

Cystinosis has three major clinical presentations: the most serious form, the 
infantile nephropathic form or classical nephropathic cystinosis (MIM 219800), a less severe form, the juvenile nephropathic form (MIM 219900) and the ocular non-nephropathic form (MIM 219750) [18]. The infantile or classical cystinosis, comprising about $95 \%$ of all cases. The non-nephropathic, or ocular cystinosis manifests exclusively with ocular involvement due to cystine crystals deposition [18] [19] [20]. In some patients, ocular involvement can precede renal manifestation by several years [21]. It has been hypothesized that the underlying differences between the clinical forms and the severity of them is because individuals with infantile cystinosis harbour "severe" mutations (i.e. loss of function) on both alleles, whereas individuals with milder forms of cystinosis harbour a specific, non-severe mutation (i.e. never observed in cases of infantile cystinosis) either on both alleles or in association with a severe allele (compound heterozygous) that is why is so important to characterized the mutation spectrum of this disease [7] [22] [23] [24]. Molecular analysis of the CTNS gene also confirms the diagnosis and offers the advantage of a prenatal diagnosis [25]. Several lines of treatment are available for cystinosis. When started at an early age, cysteamine treatment prevents or postpones the deterioration of renal function and the occurrence of extra-renal complications such as hypothyroidism, diabetes mellitus, retinopathy, encephalopathy and myopathy [26] [27].

The aim of this study was to determine the mutational spectrum of Portuguese population for cystinosis, as well as established a genotype/phenotype correlation to the mutation found with the respective cystine intra-leucocytes value and the clinical presentation. This study is the first one in the Portuguese population.

\section{Material and Methods}

\subsection{Patients}

Studies were conducted from 1998 to 2017, where a total of 1,941,372 newborns were registered. In this cohort, 32 unrelated patients from different pediatric and adult hospitals all over Portugal had non-nephrotic proteinuria $(<3.5 \mathrm{~g}$ protein/24H), hypercalciuria, hypokalemia, impaired proximal reabsorption of amino acids (hyperaminoaciduria), glycosuria and hypophosphatemia, suggestive of a Fanconi syndrome. Later, all developed also ocular complains, that revealed intraocular cystine crystals, a very important find to correlate this Fanconi syndrome with cystinosis. Although most patients had Fanconi syndrome (20/21), one patient had no Fanconi syndrome but an earlier nephrotic chronic disease (with kidney damage and $>3.5 \mathrm{~g}$ protein $/ 24 \mathrm{H}$ ) diagnosed only in adolescence (at the age of 15 years). In this particular patient, the diagnosis of the juvenile nephropatic form of cystinosis was done when she got pregnant. In fact, from these 32 patients, 21 patients revealed high values of intra-leukocytes cystine in blood ( $\geq 0.50 \mathrm{nmol} 1 / 2$ cystine/mg protein). Unfortunately, from these 21 patients ( 8 males, and 13 females, with ages between 1 - 40 years), four deceased, two we lost the clinical history (i.e. emigration) and from the remaining fifteen, 
only eleven were characterized at a molecular level. From this study, it seems that generally, not only ocular cystine crystals preceded Fanconi syndrome, but also contributes to the correct final diagnosis. The authors would like to emphasize, that from these 21 positive cystinosis patient, only one patient was diagnosed in adulthood (at the age of 40 years old) due to presence of intraocular cystine crystals, although he have had chronic nephrotic complains (such as proteinuria, calciuria, hypokalemia and hyperaminoaciduria) since pediatric age.

\subsection{Ethics Statement}

All procedures were followed in accordance with the ethical standards of the responsible committee on human experimentation (institutional and national) and with the Helsinki Declaration of 1975, as revised in 2000 and approved by the Ethics Committees. Informed consent was obtained from all patients and their families for being included in the study.

\subsection{Biochemical Methods}

The specific biochemical diagnosis was performed by study of urine and the detection of elevated intra-leukocyte cystine levels (Whole Blood Cells Cystine intra-leukocyte levels; WBC Cis IL) [28]. Prior extraction, cystine quantification was obtained by ion exchange liquid chromatography in a BIOCHROM $30^{\circ}$. The reference values used were:

- Healthy individual: $<0.5 \mathrm{nmol} 1 / 2$ cystine/mg protein.

- Affected individuals without treatment: $\geq 0.5 \mathrm{nmol} 1 / 2$ cystine/mg protein.

- Individuals treated with good therapeutic control: $\leq 1.0 \mathrm{nmol} 1 / 2$ cystine/mg protein.

\subsection{Molecular Diagnosis}

Genomic DNA was automatically extracted from whole blood or dried blood spots, using an automated method (EZ1 DNA Blood $350 \mu$ l, or EZ1 DNA tissue kit, QIAGEN). The ten protein-coding exons and flanking intronic sequences of CTNS gene (NM_004937) were directly sequenced after PCR amplification in an ABI PRISM ${ }^{\mathrm{TM}}$ 3130XL Genetic Analyzer (Applied Biosystems, Foster City, CA, USA) [7]. All patients were first screened for the 57-kb and if negative, whole $C T N S$ gene was sequenced. As a control population, we studied 100 healthy (200 alleles) unrelated individuals of Portuguese origin.

\section{Results and Discussion}

During 19 years, 32 patients with non-nephrotic proteinuria, hypercalciuria, hypokalemia, hyperaminoaciduria, glycosuria and hypophosphatemia, suggestive of a renal Fanconi syndrome and/or ophthalmologic symptoms were biochemically evaluated due to cystinosis clinical suspicion. Usually the diagnosis is made shortly after the onset of the symptoms but some delay may occur due to the low specificity of the symptoms. From those 32 patients suspected, 11 had an 
intra-leukocytes cystine value below $0.5 \mathrm{nmol} 1 / 2$ cystine/mg protein in two successive samples [4] and they were considered negative for cystinosis and other causes were investigated. In the remaining 21 , intra-leukocytes cystine ranging from 0.5 to $8.0 \mathrm{nmol} \mathrm{1/2} \mathrm{cystine/mg} \mathrm{protein} \mathrm{was} \mathrm{confirmed} \mathrm{and} \mathrm{eleven} \mathrm{of}$ these were studied for CTNS gene mutations, four pathogenic reported CTNS mutations were detected, being the common European $57-\mathrm{kb}$ deletion the most frequent (68.2\%; 15/22 alleles). Five out of eleven patients were homozygous for the $57-\mathrm{kb}$ deletion, and five were compound heterozygous for $57-\mathrm{kb}$ deletion (45.5\%) and p.Q128X (c.721 C>T; 9.1\%), p.S139F (c.755 C>T; 9.1\%) and c.18-21delGACT (p.T7FfsX7; 4.5\%), mutations (Table 1). One patient was homozygous for p.S139F mutation. From the literature, it seems that this mutation in homozygous state is the first time found. We can also conclude that patients with severe or truncating mutations, as the $57-\mathrm{kb}$ deletion and p.T7FfsX7 or p.Q128X had the infantile severe form of the disease, while juvenile cystinosis was associated with at least one mild mutation. We found that patients with the $57-\mathrm{kb}$ deletion had higher cystine intra-leukocyte levels (WBC Cis IL), which is also in concordance with the literature (Table 1), because this specific mutation results in the absence of protein [4] [8]. In Portugal, it is estimated that this disease has an incidence of 1/92,000 newborns.

The most common CTNS mutation in Northern Europe is the 57-kb deletion, which is in accordance with our results. In fact, the $57-\mathrm{kb}$ deletion accounts for about $50 \%$ of cystinosis chromosomes in European populations [29] [30]. It is thought that this deletion has arisen because of a founder effect, originated in Germany around the middle of the first millennium A.D. [3] [7] [9]. The hypothesis of this deletion having arisen in the Northern Europe and having spread throughout Europe toward Northern Africa by migration is consistent with a north to south gradient as already found for the p.F508del cystic fibrosis mutation. In fact, African influence is largely concentrated in the Southern and Western regions of Europe, and Germanic influence is small and limited. Therefore, when comparing our results with other countries from North European and North American origin, the 57-kb deletion in Portugal is also the most prevalent mutation $(68.2 \%$ of the mutant alleles). This is also true, for other countries, as Spain [12], which has a $57-\mathrm{kb}$ deletion prevalence of $34 \%$, in Netherlands [31] 59\% and 65\% in Switzerland-Germany population [11], but it decreases to $17 \%$ in Italy [14] and is absent in Turkish population. At a first glance, we should expect to have a cystinosis spectrum mutation similar to the Southern Europe population (i.e. due to our emigration history), but in fact does not happen. We have a different mutational spectrum for CTNS gene comparing with the mutational spectrum of African, Mediterranean and Middle East populations. Indeed, it seems that outside this geographical distribution (North Europe and North America), the 57-kb deletion is almost absent, especially in the Middle East [32] which appears instead to have a common exonic splice site mutation; p.E227E (c.681 G>A) [33]. In fact, this mutation, comprises $39.5 \%$ of the Iranian [15], 20\% of Turkish [34] and 15.4\% of Saudi familial mutant alleles [33] 
Table 1. Correlation between the genotype, value of intra-leukocyte cystine at the age of diagnosis and presentation form of cystinosis in the studied patients.

\begin{tabular}{cccc}
\hline Mutation Status & Patients & $\begin{array}{c}\text { Intra-Leukocyte Cystine Value } \\
\text { at Diagnosis } \\
(\text { nmol1/2cystine/mg protein })\end{array}$ & $\begin{array}{c}\text { Presentation } \\
\text { Form of Cystinosis }\end{array}$ \\
\hline 57-kb deletion/57-kb deletion & $(\mathrm{N}=5)$ & {$[2.8-8.0]$} & $\begin{array}{c}\text { Infantile } \\
\text { Nephropathic form } \\
\text { Infantile }\end{array}$ \\
57-kb deletion/Q128X & $(\mathrm{N}=2)$ & {$[1.5$ and 5.1] } & $\begin{array}{c}\text { Nephropathic form } \\
\text { Infantile }\end{array}$ \\
p.S139F/p.S139F & $(\mathrm{N}=1)$ & {$[7.3]$} & $\begin{array}{c}\text { Nephropathic form } \\
\text { Juvenile (late) }\end{array}$ \\
& $(\mathrm{N}=1)$ & {$[1.7]$} & Nephropathic form \\
57-kb deletion/S139F & $(\mathrm{N}=2)$ & {$[2.2$ and 3.7] } & $\begin{array}{c}\text { Juvenile } \\
\text { Nephropathic form }\end{array}$ \\
\hline
\end{tabular}

and was not detected previously in European or American populations. So, it seems that toward to Mediterranean region, the frequency of the $57-\mathrm{kb}$ deletion decreases. Nevertheless, the 57-kb deletion should be the first mutation for looking for in cystinosis patients from Portugal or as well as from Spain [4]. The other mutations; p.S139F as well as the c.18-21delGACT (p.T7FfsX7), have a Spanish founder gene [12], but an interesting fact is that, the deletion c.18-21delGACT (p.T7FfsX7) has been found in different populations from Europe to Middle East, and with widely disease severities. From the cohort studied, we do not have none ocular non-nephropathic form of cystinosis (Table 1), that could be related to the rarity of that form of the disease.

We suggest that this fact is due to the difficulty of established this diagnosis and most ophthalmologists do not suspect of this disease. The prognosis of cystinosis depends on early diagnosis and or the prompt start and good compliance with cysteamine treatment. From our point of view, it is important to reinforce the need to diagnosis cystinosis as earlier as possible, and begin therapy with cystine-depleting agents as soon as the diagnosis is made. In this way, it may be possible to attenuate the renal tubular Fanconi syndrome and significantly slow the progression of glomerular damage. Kidney disease progression, extra-renal complications and shorter life expectancy are more evident in patients who do not adhere to treatment.

\section{Conclusions}

Four different mutations were identified: one large deletion (57-kb deletion), one small deletion (c.18-21delGACT) that originated a frameshift mutation (p.T7FfsX7), one missense mutation (p.S139F) and one nonsense mutation that originates a premature stop codon (p.Q128X). The $57-\mathrm{kb}$ deletion is the most common mutation found in the Portuguese patients (68.2\%).

The authors would like to emphasize the importance of first screening for the 
57-kb deletion since it is very common in our population, although the determination of intra-leukocytes cystine (WBC Cis IL) is also an important tool for the cystinosis diagnosis and therapeutic monitorization. Although, it is important to notice that in small infants, WBC Cis IL may result in a false negative. This genetic study is the first in our country and it could be the basis for future genetic counseling and prenatal diagnosis of patients with nephropathic cystinosis in Portuguese population. Prenatal diagnosis allows for early cysteamine treatment. We also like to emphasize that, to those countries with high rate of consanguinity, the incidence of cystinosis is predicted to be higher, we should suggest in a potential universal neonatal screening for cystinosis.

\section{Take Home Message}

Mutational spectrum of Portuguese population for cystinosis. This study is the first one in the Portuguese population.

\section{Compliance with Ethical Standards}

The submitting author confirms that all procedures were followed in accordance with the ethical standards of the responsible committee on human experimentation (institutional and national) and with the Helsinki Declaration of 1975, as revised in 2000 and approved by the Ethics Committees.

\section{Informed Consent}

Informed consent was obtained from all patients and/or their families for being included in the study.

\section{Author's Contribution}

Filipa Ferreira and Laura Vilarinho contributed to all aspects of the study manuscript. Filipa Ferreira involved in the conception, conducting, interpreting of data, drafting the manuscript and revising it critically. Laura Vilarinho involved in planning, revising the manuscript critically for important intellectual content and final approval of the version to be submitted. Célia Carmona; Sónia Ramos; Raquel Neiva, Inês Leal, David Sousa, Teresa Costa, Conceição Mota, Ana Marta Gomes, Daniela Lopes, Maria do Carmo Macário, Isabel Tavares, Helena Pinto, João Paulo Oliveira, Rita Magriço involved in acquisition of data and reviewed manuscript and revising it critically for important intellectual content.

\section{Funding}

This research did not receive any specific grant from funding agencies in the public, commercial, or not-for-profit sectors.

\section{Acknowledgements}

Authors are thankful to Dr Francisco Pérez Rodríguez and Doctor Eduardo Alonso. 


\section{Conflicts of Interest}

The authors declare no conflicts of interest regarding the publication of this paper.

\section{References}

[1] Abderhalden, E. (1903) Familiare Cystindiathese. Hoppe-Seyler's Zeitschrift für physiologische Chemie, 38, 557-561. https://doi.org/10.1515/bchm2.1903.38.5-6.557

[2] Town, M., Jean, G, Cherqui, S., Attard, M., Forestier, L., Whitmore, S.A., Callen, D.F., Gribouval, O., Broyer, M., Bates, G.P., van't Hoff, W. and Antignac, C. (1998) A Novel Gene Encoding an Integral Membrane Protein Is Mutated in Nephropathic Cystinosis. Nature Genetics, 18, 319-324. https://doi.org/10.1038/ng0498-319

[3] Gahl, W.A., Thoene. J.G. and Schneider, J.A. (2002) Cystinosis. The New England Journal of Medicine, 347, 111-121. https://doi.org/10.1056/NEJMra020552

[4] Ariceta, G., Camacho, J.A., Fernández-Obispo, M., et al. (2015) Cistinosisenpacienties adolescentes y adultos: recomendaciones para la atención de la cistinosis. $\mathrm{Ne}$ frologia, 35, 304-321.

[5] Nesterova, G. and Gahl, W.A. (2014) Cystinosis. In: Adam, M.P., Ardinger, H.H., Pagon, R.A., et al., Eds., Gene Reviews, University of Washington, Seattle, 1993-2018.

[6] Levtchenko, E., de Graaf-Hess, A., Wilmer, M., van den Heuvel, L., Monnens, L. and Blom, H. (2004) Comparison of Cystine Determination in Mixed Leukocytes vs Polymorphonuclear Leukocytes for Diagnosis of Cystinosis and Monitoring of Cysteamine Therapy. Clinical Chemistry, 50, 1686-1688. https://doi.org/10.1373/clinchem.2004.031872

[7] Anikster, Y., Lucero, C., Touchman, J.W., Huizing, M., McDowell, G., Shotelersuk, V., et al. (1999) Identification and Detection of the Common 65-kb Deletion Breakpoint in the Nephropathic Cystinosis Gene (CTNS). Molecular Genetics and Metabolism, 66, 111-116. https://doi.org/10.1006/mgme.1998.2790

[8] Touchman, J.W., Anikster, Y., Dietrich, N.L., Maduro, V.V., McDowell, G., Shotelersuk, V., et al. (2000) The Genomic Region Encompassing the Nephropathic Cystinosis Gene (CTNS): Complete Sequencing of a 200-kb Segment and Discovery of a Novel Gene within the Common Cystinosis-Causing Deletion. Genome Research, 10, 165-173. https://doi.org/10.1101/gr.10.2.165

[9] Forestier, L., Jean, G., Attard, M., et al. (1999) Molecular Characterization of CTNS Deletions in Nephropathic Cystinosis: Development of a PCR-Based Detection Assay. American Journal of Human Genetics, 65, 353-359. https://doi.org/10.1086/302509

[10] Anikster, Y., Lucero, C., Guo, J., et al. (2000) Ocular Non-Nephropathic Cystinosis: Clinical, Biochemical, and Molecular Correlations. Pediatric Research, 47, 17-23. https://doi.org/10.1203/00006450-200001000-00007

[11] Kiehntopf, M., Schickel, J., Gönne, B.V., Koch, H.G., Superti-Furga, A., Steinmann, B., Deufel, T. and Harms, E. (2002) Analysis of the CTNS Gene in Patients of German and Swiss Origin with Nephropathic Cystinosis. Human Mutation, 20, 237. https://doi.org/10.1002/humu.9063

[12] Macías Vidal, J., Rodés, M., Hernández-Pérez, J.M., et al. (2009) Analysis of the CTNS Gene in 32 Cystinosis Patients from Spain. Clinical Genetics, 76, 486-489. https://doi.org/10.1111/j.1399-0004.2009.01222.x

[13] Chkioua, L., Khedhiri, S., Grissa, O., et al. (2015) Genetic Basis of Cystinosis in Tu- 
nisian Patients: Identification of Novel Mutation in CTNS Gene. Meta Gene, 25, 144-149. https://doi.org/10.1016/j.mgene.2015.07.003

[14] Mason, S., Pepe, G., Dall'Amico, R., et al. (2003) Mutational Spectrum of the CTNS Gene in Italy. European Journal of Human Genetics, 11, 503-508. https://doi.org/10.1038/sj.ejhg.5200993

[15] Shahkarami, S., Galehdari, H., Ahmadzadeh, A., et al. (2013) The First Molecular Genetics Analysis of Individuals Suffering from Nephropatic Cystinosis in the Southwestern Iran. Nefrologia, 33, 308-315.

[16] Ghazi, F., Hosseini, R., Akouchekian, M., Shahram, T., Zohreh, A.K., Hassan, O., William, A.G. and Babak, B. (2017) CTNS Molecular Genetics Profile in a Persian Nephropathic Cystinosis Population. Nefrologia, 37, 301-310. https://doi.org/10.1016/j.nefro.2016.11.024

[17] Kalatzis, V., Nevo, N., Cherqui, S., et al. (2004) Molecular Pathogenesis of Cystinosis: Effect of CTNS Mutations on the Transport Activity and Subcellular Localization of Cystinosin. Human Molecular Genetics, 13, 1361-1371. https://doi.org/10.1093/hmg/ddh152

[18] Gahl, W.A., Thoene, J. and Schneider, J. (2001) Cystinosis: A Disorder of Lysosomal Membrane Transport. In: Scriver, C.J., Beaud, Sly, W.S. and Valle, D., Eds., The Metabolic and Molecular Bases of Inherited Disease, McGraw-Hill, New York, 5085-5108.

[19] Nesterova, G. and Gahl, W.A. (2008) Nephropathic Cystinosis: Late Complications of a Multisystemic Disease. Pediatric Nephrology, 23, 863-867.

[20] Pintos, G. (2011) Cystinosis: From Cystine Crystals to the Cystinosin. Nefrologia, 23, 60-70.

[21] Servais, A., Morinière, V., Grünfeld, J.P., et al. (2008) Late-Onset Nephropathic Cystinosis: Clinical Presentation, Outcome, and Genotyping. Clinical Journal of the American Society of Nephrology, 3, 27-35. https://doi.org/10.2215/CJN.01740407

[22] Attard, M., Jean, G., Forestier, L., et al. (1999) Severity of Phenotype in Cystinosis Varies with Mutations in the CTNS Gene: Predicted Effect on the Model of Cystinosin. Human Molecular Genetics, 8, 2507-2514. https://doi.org/10.1093/hmg/8.13.2507

[23] Thoene, J., Lemons, R., Anikster, Y., et al. (1999) Mutations of CTNS Causing Intermediate Cystinosis. Molecular Genetics and Metabolism, 67, 283-293. https://doi.org/10.1006/mgme.1999.2876

[24] Kalatzis, V. and Antignac, C. (2002) Cystinosis: From Gene to Disease. Nephrology Dialysis Transplantation, 17, 1883-1886. https://doi.org/10.1093/ndt/17.11.1883

[25] Nesterova, G. and Gahl, W.A. (2013) Cystinosis: The Evolution of a Treatable Disease. Pediatric Nephrology, 28, 51-59. https://doi.org/10.1007/s00467-012-2242-5

[26] Elmonem, M.A., Veys, K.R., Soliman, N.A., et al. (2016) Cystinosis: A Review. Orphanet Journal of Rare Diseases, 11, 47. https://doi.org/10.1186/s13023-016-0426-y

[27] Ariceta, G., Giordano, V. and Santos, F. (2017) Effects of Long-Term Cysteamine Treatment in Patients with Cystinosis. Pediatric Nephrology, 1-8. https://doi.org/10.1007/s00467-017-3856-4

[28] Schneider, J.A., Bradley, K. and Seegmiller, J.E. (1967) Increased Cystine in Leukocytes from Individuals Homozygous and Heterozygous for Cystinosis. Science, 157, 1321-1322. https://doi.org/10.1126/science.157.3794.1321

[29] Shotelersuk, V., Larson, D., Anikster, Y., et al. (1998) CTNS Mutations in an American-Based Population of Cystinosis Patients. American Journal of Human Genet- 
ics, 63, 1352-1362. https://doi.org/10.1086/302118

[30] Levtchenko, E., van den Heuvel, L., Emma, F., et al. (2014) Clinical Utility Gene Card for: Cystinosis. European Journal of Human Genetics, 22, 1-3.

https://doi.org/10.1038/ejhg.2013.204

[31] Heil, S.G., Levtchenko, E., Monnens, L.A.H., Trijbels, F.J.M., van der Put, N.M.J. and Blom, H.J. (2001) The Molecular Basis of Dutch Infantile Nephropathic Cystinosis. Nephron, 89, 50-55. https://doi.org/10.1159/000046043

[32] Soliman, N.A., Elmonem, M.A., van den Heuvel, L., et al. (2014) Mutational Spectrum of the CTNS Genein Egyptian Patients with Nephropathic Cystinosis. JIMD Reports, 14, 87-97. https://doi.org/10.1007/8904_2013_288

[33] Aldahmesh, M.A., Humeidan, A., Almojalli, H.A., et al. (2009) Characterization of CTNS Mutations in Arab Patients with Cystinosis. Ophthalmic Genetics, 30, 185-189. https://doi.org/10.3109/13816810903200953

[34] Topaloglu, R., Vilboux, T., Coskun, T., et al. (2012) Genetic Basis of Cystinosis in Turkish Patients: A Single-Center Experience. Pediatric Nephrology, 27, 115. https://doi.org/10.1007/s00467-011-1942-6 